\title{
Small Incision Cataract Surgery (SICS) with Posterior Chamber Intraocular Lens (PCIOL) Implantation is the Preferred Method of Community-Based Cataract Surgery: Study of 100 Cases
}

\author{
A K M Shahidur Rahman Tarafder ${ }^{1}$, M Anwarul Kader ${ }^{2}$, S M Rezaul Karim ${ }^{3}$
}

\begin{abstract}
To study "small incision cataract surgery (SICS)" for the rehabilitation of cataract visually impaired and blind patients, a retrospective study of 100 cases of cataract patients with small incision cataract surgery (SICS) with posterior chamber intraocular lens (PCIOL) implantation were done at Rajshahi Medical College Hospital. All cases were selected having corrected visual acuity less than 6/60 who were admitted at the Department of Ophthalmology for cataract surgery from March, 2006 to February, 2008. Age group of the patient range from 40 to 85 years. Age related senile cataract were selected any complicated cataract was excluded from the study. All these patients underwent SICS with $5.5 \mathrm{~mm}$ optic polymethylmethacrylate (PMMA) non-foldable posterior chamber intraocular lens (PCIOL) were implanted by irrigation vectis. Intra operative complication were present in $8 \%$ cases among them 3 cases (3\%) required to conversion to Conventional Extracapsular Cataract Extraction (ECCE). 2 cases (2\%) had small posterior capsular rent (PCR), 1 case (1\%) irido dialysis \& 2 cases (2\%) hyphaema occurred. Postoperative complications were found in 38 cases (38\%) among them transient corneal oedema occurred in 18 cases (18\%) iritis occurred in 13 cases (13\%), decentering of intraocular lens (IOL) occurred in 2 cases, corrected visual acuity after 6 weeks of operation were 6/12 or better in 97 cases ( $97 \%$ cases achieved functional vision). Induced astigmatism $\pm 0.50 \mathrm{D}$ to \pm $1.00 \mathrm{D}$ occurred in 94 cases (94\%) and \pm 1.500 to $2.50 \mathrm{D}$ in 6 cases $(6 \%)$. So, SICS may be the choice of surgery in respect of visual outcome with the limited facilities and surgeons.
\end{abstract}

TAJ 2009; 22(1): 132-135

\section{Introduction}

Among the methods of cataract surgery i.e. conventional ECCE, Small incision cataract surgery (SICS) and cataract extraction by phaco emulsification. Small incision cataract surgery (SICS) is a good alternative to phaco emulsification in countries where very high volume surgery with inexpensive instrument is required ${ }^{1}$. It is the best method of community based operation, having less cost effectiveness and early rehabilitation of the patient ${ }^{2}$. In Bangladesh only about 500 ophthalmologists are engaged to eliminate a huge backlog of 1.5 million cataract patient. Unfortunately with all efforts yearly 80,000 to $1,00,000$ surgeries are performed. This number is almost equal to new cases that are added yearly to the backlog. If this is going on, the number of cataract patient will be double in another 50 to 20 years $^{3}$. SICS began the modern day with three step (or three plane) internal

${ }^{1}$ Associate Professor, Department of Ophthalmology, Rajshahi Medical College, Rajshahi.

${ }^{2}$ Assistant Professor, Department of Ophthalmology, Rajshahi Medical College, Rajshahi.

${ }^{3}$ Assistant Professor, Department of Ophthalmology, Rajshahi Medical College, Rajshahi. 
corneal lip incision also known as the corneal valve incision, which incorporates a perpendicular incision through the sclera, a horizontal incision into clear cornea and then angled beveled incision in the anterior chamber. This three steps procedure leaves an internal lip of endothelium, descemets membrane and corneal stroma that seals on itself when the intraocular pressure returns to normal ${ }^{4}$. There is no stitch required \& minimum time (2025 minutes) is required to perform the operation. So the study was done to see whether SICS with PCIOL is the choice of surgery in respect to visual out come, cost effectiveness and minimum surgical time to over come the backlog with the limited facilities of instruments and surgeon.

\section{Materials \& Methods}

Patients were selected from those who were admitted for cataract surgery at Department of Ophthalmology, Rajshahi Medical College Hospital from March, 2006 to February, 2008, 100 cases of age related senile cataract were selected having corrected visual acuity less than 6/60. All the cases were age related cataract (no complicated cataract included). 57 cases (57\%) are female and 43 (43\%) were male patient, age ranged from 40 to 85 years. Routine preoperative investigation was done, pupil were dilated with tropicamide $1 \%$ and phenylephrin $2.5 \%$ eye drop. Acetazolamide $250 \mathrm{mg}$ tablet were given 2 hours prior to surgery. For corneal \& conjunctival anaesthesia oxybuprocaine $0.4 \%$ was instilled five times with one minute interval before giving peribulbar block. All surgeries were done under local anaesthesia.

\section{Procedure}

Informed consent was taken and peribulbar block (4ml of inj. Lignocaine $2 \%$ plus $4 \mathrm{ml}$ of inj. Bupivacin $0.5 \%$ plus inj. Hyaluronidase (10 turbidity unit per ml) was given. Massage with guaze piece and finger over closed eye lid was applied for 5 minutes. Eye and surrounding areas was wetted with povidone iodine (5\% in aqueous solution) then eye was drapped. After applying speculum and holding superior rectus fornix based conjunctival flap was fashioned. Thromocautery used carefully to prepare a blood less field to operate. A straight incision of 7-8 $\mathrm{mm}$ was made
$1.5 \mathrm{~mm}$ behind the limbus by crescent knife scleral tunnel was made in such a way that it was 10 to 11 $\mathrm{mm}$ internally (by extending scleral pocket towards the corneal stroma). The corneal tunnel was made $1.5 \mathrm{~mm}$ from the limbus, incase of harder nucleus the incision was extended. Entry into the anterior chamber is made with $3.2 \mathrm{~mm}$ angled keratome. Anterior capsule stained by Trypan blue, visco elastic substance (hydroxy propyl methyl cellulose) was introduced into the anterior chamber. A side port was made at the temporal side, capsulorrhexis was done then after hydrodissection, breaking all the adhesion of nucleus within the bag, two relaxing incision at the edge of the capsulorhesix at 11 o'clock and 2 o'clock position were given to facilitate the prolapse of the nucleus to anterior chamber from the capsular bag. The tunnel was enlarged 7-8 mm externelly and 11 to $12 \mathrm{~mm}$ internally viscoelastic substance was introduced above and below the nucleus. The nucleus was taken out by irrigating vectis, the cortical matter was washed through the main wound and side port by simco two way irrigation aspiration cannula, then viscoelastic substance is introduced into the anterior chamber and $5.5 \mathrm{~mm}$ optic PMMA non foldable PCIOL was implanted at capsular bag, the viscoelastic substance was washed out by the same cannula. Conjunctiva was reposited back by holding the conjunctiva with forcep, cautery was applied at two ends. Then gentamycin and Dexamethason subconjunctival injection was given. Ocular pad was applied, all patients are followed on $1^{\text {st }}$ and $2^{\text {nd }}$ post operative day and 6 weeks after operation.

\section{Results}

Age distribution of the patient selected for SICS with PCIOL implantation ranged from 40 to 85 years.

\section{Complications}

Intraoperative complications occurs in 08 cases $08 \%$ incision was required to enlarge and conversion of the procedure to ECCE was done in 4 cases (4\%) (due to hard \& large nucleus 8-9 mm in 3 cases \& premature entry at anterior chamber in 1 case $(1 \%)$ during scleral tunnel. Posterior capsular rent occurred in 3 cases (3\%), irodiodialysis in 1 case (1\%) and hyphaema occurred in 2 cases (2\%) Table-1. 
Table-1: Intraoperative complications of SICS.

\begin{tabular}{|c|c|c|}
\hline Type of Complication & $\begin{array}{c}\text { No. of } \\
\text { patient }\end{array}$ & Percent \\
\hline \multicolumn{3}{|l|}{ 1. Enlargement of incision (ECC) } \\
\hline (a) Large \& Hard nucleus 8-9mm & 03 & $03 \%$ \\
\hline (b) Pre mature entry in AC & 01 & $01 \%$ \\
\hline $\begin{array}{l}\text { 2. Posterior capsular rent (PCR): } \\
\text { With vitrous prolaps }\end{array}$ & 01 & $01 \%$ \\
\hline 3. Irido dialysis & 01 & $01 \%$ \\
\hline 4. Hyphaema & 02 & $02 \%$ \\
\hline Total= & 08 & $08 \%$ \\
\hline
\end{tabular}

\section{Post operative complications}

Occurred in 38 cases (38\%) transient corneal oedema occurred in 18 cases (18\%), iritis occurred in 13 cases (13\%) among them moderate iritis 11 cases (11\%) and severe iritis in 2 cases (2\%). Decentering of PCIOL occurred in to 2 cases (2\%) posterior capsular opacification of different densities occurred in 5 cases (5\%). Table-2.

Table-2: Postoperative complication.

\begin{tabular}{lcc}
\hline \multicolumn{1}{c}{ Complication } & $\begin{array}{c}\text { No of } \\
\text { patient }\end{array}$ & Percent \\
\hline 1. Tansient corneal oedema & 18 & $18 \%$ \\
2. Irites & 00 & 00 \\
(a) Moderate & 11 & $11 \%$ \\
(b) Severe & 02 & $02 \%$ \\
3. Decentering of PCIOL & 02 & $02 \%$ \\
4. Posterior capsular & 05 & $05 \%$ \\
$\quad$ opacification of & & \\
$\quad$ different densities. & $\mathbf{3 8}$ & $\mathbf{3 8 \%}$ \\
\hline \multicolumn{1}{c}{ Total $=$} \\
\hline
\end{tabular}

Visual acuity of SICS patient were $6 / 60$ or less in 5 cases (5\%) 6/36 to 6/18 in 71 cases (71\%), 6/12 to $6 / 9$ in 24 cases $(24 \%)$ on $1^{\text {st }}$ postoperative day and final corrected visual acuity after 6 weeks of operation were $6 / 60$ or less in 2 cases (2\%), 6/36 to $6 / 18$ in 1 case (1\%) 6/12 to 6/9 in 25 cases (25\%) and 6/6 72 cases (72\%). (Table-3)

Table-3: Visual outcome of SICS with PCIOL.

\begin{tabular}{lcccc}
\hline Visual & $\begin{array}{c}\mathbf{1}^{\text {st }} \text { POD, } \\
\text { Number of } \\
\text { Patient with } \\
\text { Uncorrected } \\
\text { VA }\end{array}$ & Percent & $\begin{array}{c}\text { 6 weeks after } \\
\text { operation } \\
\text { number of } \\
\text { patient with } \\
\text { corrected VA }\end{array}$ & Percent \\
\hline $6 / 60$ or less & 05 & $05 \%$ & 02 & $02 \%$ \\
$6 / 36$ to $6 / 18$ & 71 & $71 \%$ & 01 & $01 \%$ \\
$6 / 12$ to $6 / 9$ & 24 & $24 \%$ & 25 & $25 \%$ \\
$6 / 6$ & 00 & 00 & 72 & $72 \%$ \\
\hline \multicolumn{1}{r}{ Total $=$} & $\mathbf{1 0 0}$ & $\mathbf{1 0 0} \%$ & $\mathbf{1 0 0}$ & $\mathbf{1 0 0 \%}$ \\
\hline
\end{tabular}

Induced astigmatism correction required after 6 weeks operation was $\pm 0.5 \mathrm{D}$ to $\pm 1.00 \mathrm{D}$ in 96 cases $(96 \%)$ and $\pm 2.00 \mathrm{D}$ to $\pm 2.5 \mathrm{D}$ in 4 cases (4\%). (Table-4)

Table-4: Induced astigmatism after 6 weeks of operation.

\begin{tabular}{rcc}
\hline $\begin{array}{c}\text { Amount of } \\
\text { astigmatism }\end{array}$ & $\begin{array}{c}\text { Number of } \\
\text { patient }\end{array}$ & Percent \\
\hline $\pm 0.5 \mathrm{D}$ to $\pm 1.00 \mathrm{D}$ & 96 & $96 \%$ \\
$\pm 2.00 \mathrm{D}$ to $\pm 2.5 \mathrm{D}$ & 04 & $04 \%$ \\
\hline Total $=$ & $\mathbf{1 0 0}$ & $\mathbf{1 0 0 \%}$ \\
\hline
\end{tabular}

\section{Discussion}

Total 100 patients with PCIOL implanted by manual SICS with $5.5 \mathrm{~mm}$ to $6.00 \mathrm{~mm}$ optic size, nonfoldable PMMA were followed up in this retrospective study. The age ranging from 40-55 years. The results showed that the functional vision were achieved of 97 cases (97\%) of the patients. Intraoperative complications were developed in 8 cases (8\%) among them incision was required to enlarge in 4 cases (4\%) because large size hard nucleus $(8-9 \mathrm{~mm})$ in 3 cases $(3 \%)$ and premature entry at the anterior chamber by crescent knife during sclero-corneal tunnel construction in one case all these four cases are managed by conversion of SICS to conventional ECCE. Posterior capsular rent with vitrous prolapse occurred in 1 case. Aspiration of vitrous done by cutting the vitrous by venous scissor, clear the AC and sclearal fixation was done. In 2 cases (2\%) hyphaema occurred from the wound due to insufficient thermo cautery. In those case viscogel was introduced to $\mathrm{AC}$ and then bleeding was stopped. Post operative complications were developed in 38 cases (38\%) among them transient corneal oedema occurred in 18 cases (18\%) but were subsided by using topical steroid, oral steroid and topicamide 1\% within 7-14 days. Iritis were developed in 13 cases (13\%) among them moderate iritis in 11 cases (11\%) and severe irits in 2 cases (2\%) were managed by systemic steroid along with topical steroid and atropine within 2 weeks, all the signs were subsided. PCIOL decentring occurs in 2 cases (2\%) among them in 
1 case (1\%) PCIOL explanted and exchange with ACIOL after 6 weeks of operation. But functional vision was not regained. In this study 72 cases (72\%) had corrected vision 6/6 Visual acuity of SICS patients in $1^{\text {st }}$ operative day of was $6 / 60$ or less or in 5 cases (5\%) 6/36-6/18 in 71 cases (71\%), 6/12-6/9 in 24 cases (24\%) and final corrected visual acuity after 6 weeks of operation (after correction by refraction) was less by $6 / 60$ or less in 2 cases (2\%), 6/36-6/18 1 case (1\%), 6/12$6 / 9$ in 25 cases (25\%) and 6/6 in 72 cases (72\%) visual acuity of the displace PCIOL replace by ACLOL were not improve, so corrected visual acuity 6/12 or better (functional vision) were recorded in $97 \%$ of the cases. Regarding astigmatism in 96 cases (96\%) had $\pm 0.50 \mathrm{D}$ to \pm $1.00 \mathrm{D}$ and in 4 cases ( $4 \%$ ) had $\pm 2.00 \mathrm{D}$ to $\pm 2.50 \mathrm{D}$ was developed 6 weeks after operation. A study by Chowdhry $\mathrm{MR}^{5}$ found with penhole $6 / 9$ or better vision in $87 \%$ cases in phaco surgery. Cost of the surgery (SICS) 30 US dollar whereas in phaco it is about 150 US dollar with IOL of same value. So, cost of SICS is very minimum in comparison with phaco surgery. The results of our study co-relates well with the another study of Dr. Md. Aminul H Akhanda at Akhando Eye Center, Kishorgonj. His findings show that corrected visual acuity at $40^{\text {th }}$ post operative day were 6/12 or better in $98 \%$ cases. Relatively small incision (less injury) clear cornea, round pupil, less AC reaction and less amount of induced astigmatism in the post operative days all the clues to the better vision in SICS $^{6}$.

\section{Conclusion}

The Small Incision Cataract Surgery (SICS) is becoming popular day by day. It is the alternative of phaco surgery, In phaco surgery, a surgeon must have adequate skill and funds to established a proper setup for phaco emulsification on the other hand SICS replacing the conventional method of cataract surgery, because in SICS small incision is given, no stitch required, non-foldable lens (cheap) is used and also there is no problem to enlarge the incision and stitch may also be applied if needed. SICS with PCIOL can be performed in an eye camp where many operations are done within short time with a small team and least facilities.

So, SICS is the preferred technique to over come the huge backlog of cataract in our country.

\section{References}

1. Jack. J. Kanski, Clinical Ophthalmology $6^{\text {th }}$ edition, P-349.

2. Highlight of ophthalmology, 2009, Vol-2 No-04, P33-34.

3. Shah Md. Bulbul Islam et al Small incision cataract surgery (SICS) need of the our. Trans ophthal. SOC. Bang. 2009; 29(1): P-12-13.

4. Chakraborti A. Dr. Verma R. Dr. Scleral Tunnel in manual phaco, Manual for IOL trainees 1997: P126-31.

5. Chowdhury MR: Pbacoemulsification with foldable lens under topical lidocaine gel: Tans. OphthaL Soc.Bang. 2000 Vol-27; No-1:P-75-61.

6. Akand. Manual small incision cataract surgery (SICS) with PCIOL implantation using irrigation cectis-study of 50 cases. Trans. Ophthal. Soc. Bang. 2001; 28(2):2001 P-22-27. 